\title{
Aneurisma de arteria pulmonar: un hallazgo incidental
}

\author{
Pulmonary artery aneurysm: an incidental finding
}

Cristhian Felipe Ramírez-Ramos ${ }^{1 *}$ ORCID, Clara Inés Saldarriaga-Giraldo², Catalina Gallego ${ }^{2}$ ORCID, Juan Sebastián Parra-Puentes ${ }^{3}$ CvLAC, Juan Manuel Torres-Restrepo4, Jorge Luis Méndez-

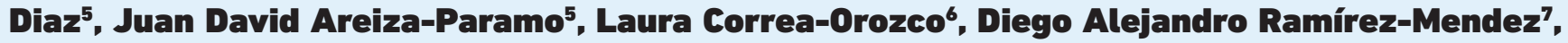
Giovanni Lastra-González ${ }^{8}$ cvLAC

Fecha correspondencia: Recibido: enero 11 de 2020. Revisado: julio 14 de 2020. Aceptado: julio 22 de 2020.

Forma de citar:

Ramírez-Ramos CF, Saldarriaga C, Gallego C, Parra-Puentes JS, Torres-Restrepo JM, Méndez-Diaz $J$ et al. Aneurisma de arteria pulmonar: un hallazgo incidental, reporte de caso y revisión de la literatura. Rev CES Med 2020; 34(2): 144-152.

Open access

(c) Derecho de autor Licencia creative commons

Ética de publicaciones

Revisión por pares

Gestión por Open Journal System DOl: http://dx.doi.org/10.21615/ cesmedicina.34.2.6

ISSN 0120-8705

e-ISSN 2215-9177

\section{Resumen}

Los aneurismas de la arteria pulmonar son entidades infrecuentes y su tratamiento es tema de discusión. Desde el punto de vista etiológico pueden ser congénitos o adquiridos. Los primeros, generalmente se asocian a malformaciones cardiacas que producen hipertensión pulmonar, siendo el ductus arterioso la más frecuente. Otras anomalías incluyen defectos auriculares o ventriculares. Las causas adquiridas pueden ser idiopáticas o estar asociadas a infecciones (tuberculosis, sífilis), traumatismos o colagenopatías. Presentamos el caso de una mujer de 62 años, quien consultó por un cuadro clínico en el que se destacaba su sintomatología neurológica e infecciosa, con posterior progresión a un choque séptico y en quien los hallazgos de las imágenes mostraron un aneurisma gigante de la arteria pulmonar siendo este un hallazgo incidental y sin relación a la sintomatología de la paciente.

Palabras clave: Aneurisma; Arteria pulmonar; Hemoptisis; Reporte de caso.

\begin{abstract}
Aneurysms of the pulmonary artery are rare entities and their treatment is a matter of discussion. From the etiological point of view, they can be congenital or acquired. Those in the first group are generally associated with cardiac malformations that generate pulmonary hypertension, with the ductus arteriosus being the most frequent. Other abnormalities include atrial or ventricular defects. The acquired causes may be idiopathic or associated with infections (tuberculosis, syphilis), trauma, or collagen disease. We present the case of a 62-year-old woman, which consulted for a clinical condition where neurological and infectious symptoms stood out, with subsequent progression to a state of septic shock, and in whom the imaging finding showed a giant pulmonary artery aneurysm. this being an incidental finding and unrelated to the patient's symptoms.
\end{abstract}

Keywords: Aneurysm; Pulmonary artery; Hemoptysis; case reports. 


\section{Sobre los autores:}

1. Médico especialista en Medicina Interna. Fellow de Cardiología Universidad Pontificia Bolivariana, Clínica CardioVID Medellín Colombia.

\section{Médica especialista en Medicina Interna y Cardiología, Universidad Pontificia Bolivariana, Universidad de Antioquia, Clínica CardioVID Medellín Colombia.}

\section{Médico especialista en} Cirugía General, Clínica Los Nogales, Bogotá Colombia. Hospital Universitario Clínica San Rafael.

\section{Residente de Cirugía General Universidad Surcolombiana y Hospital Universitario Hernando Moncaleano Perdomo, Neiva Colombia.}

\section{Médico, Hospital Departamental San Antonio, Pitalito Colombia.}

\section{Médico Sura EPS, Medellín Colombia.}

\section{Médico. Universidad} Surcolombiana, Medellín Colombia.

\section{Médico internista} neumólogo, jefe del servicio de Neumología Hospital Universitario Hernando Moncaleano Perdomo, Neiva Colombia.

\section{Introducción}

Los aneurismas de la arteria pulmonar son raros e infrecuentemente diagnosticados. Deterling y Clagett descubrieron ocho casos de aneurismas de la arteria pulmonar en 109571 en exámenes postmortem consecutivos (1). Estos, generalmente ocurren en un grupo de edad más joven, comparado con los aneurismas aórticos y tienen un incidencia igual por sexos (2). El $89 \%$ se localizan en el tronco principal de la arteria pulmonar y $11 \%$ en las ramas arteriales, siendo más frecuentes los del lado izquierdo (3).

Presentamos el caso de una paciente quien consultó por síntomas neurológicos e infecciosos y en quien se detectó aneurisma gigante del tronco de la arteria pulmonar, con el objetivo de realizar una revisión de la literatura de esta enfermedad poco frecuente. La paciente suministró el consentimiento informado para utilización de los datos en el presente reporte.

\section{Presentación del caso}

Una paciente de 62 años consultó por cuadro clínico de 20 días de evolución consistente en episodios de fluctuación del estado de alerta, con episodios de somnolencia y postración, sin focalización neurológica, estaba asociado a eventos de alucinaciones visuales, desorientación en tiempo y espacio y además hetero-agresividad. Los familiares refirieron que había presentado tos con expectoración de características no descritas y fiebre no cuantificada, sin otra sintomatología asociada. Como antecedentes contaba hipotiroidismo en suplencia hormonal e hipertensión arterial sistémica en manejo con enalapril y amlodipino.

A su llegada al servicio de urgencias presentaba presión arterial de 140/70 mm $\mathrm{Hg}$, frecuencia cardiaca de $84 \mathrm{lat} / \mathrm{min}$, frecuencia respiratoria de $18 \mathrm{resp} / \mathrm{min}$ y se detectó soplo sistólico en foco mitral grado V/VI, irradiado a la axila, con frémito. En el examen neurológico se encontró somnolencia, sin focalización motora aparente. Se realizó tomografía computarizada simple de cráneo que descartó un evento vascular encefálico agudo y los estudios analíticos no reportaron anormalidades electrolíticas o metabólicas que explicaran su cuadro (cuadro).

Pocas horas después del ingreso la paciente sufrió hipotensión arterial, sin respuesta a fluidos endovenosos, por lo que requirió vasopresores y traslado a unidad de cuidados intensivos para monitorización. Una radiografía de tórax inicial evidenció una gran radio-opacidad en el campo pulmonar derecho (figura 1) considerándose entonces posible foco infeccioso pulmonar, por lo que se inició tratamiento antimicrobiano. Debido a la gran sintomatología neurológica se decidió realizar punción lumbar. Las características del líquido cefalorraquídeo fueron normales.

Se solicitaron angiotomografía de tórax y ecocardiograma. El ecocardiograma mostró un crecimiento significativo de cavidades derechas (relación ventrículo derecho/ izquierdo $>1$ ), insuficiencia tricuspídea moderada e insuficiencia pulmonar severa con dilatación de la arteria pulmonar; hipertensión pulmonar con presión sistólica pulmonar estimada de $78 \mathrm{~mm} \mathrm{Hg}$ y cavidades izquierdas sin mayores alteraciones, con una fracción de eyección del ventrículo izquierdo preservada (58 \%). No se encontraron defectos septales.

La imagen tomográfica del tórax mostró una dilatación aneurismática sacular severa del tronco de la arteria pulmonar $(140 \mathrm{~mm}$ en diámetro mayor en corte axial y $121 \mathrm{~mm}$ en diámetro máximo en corte coronal) y de la rama derecha (44 mm), sin 
trombos en la circulación pulmonar. Las imágenes del parénquima pulmonar descartaron proceso neumónico asociado (figura 2).

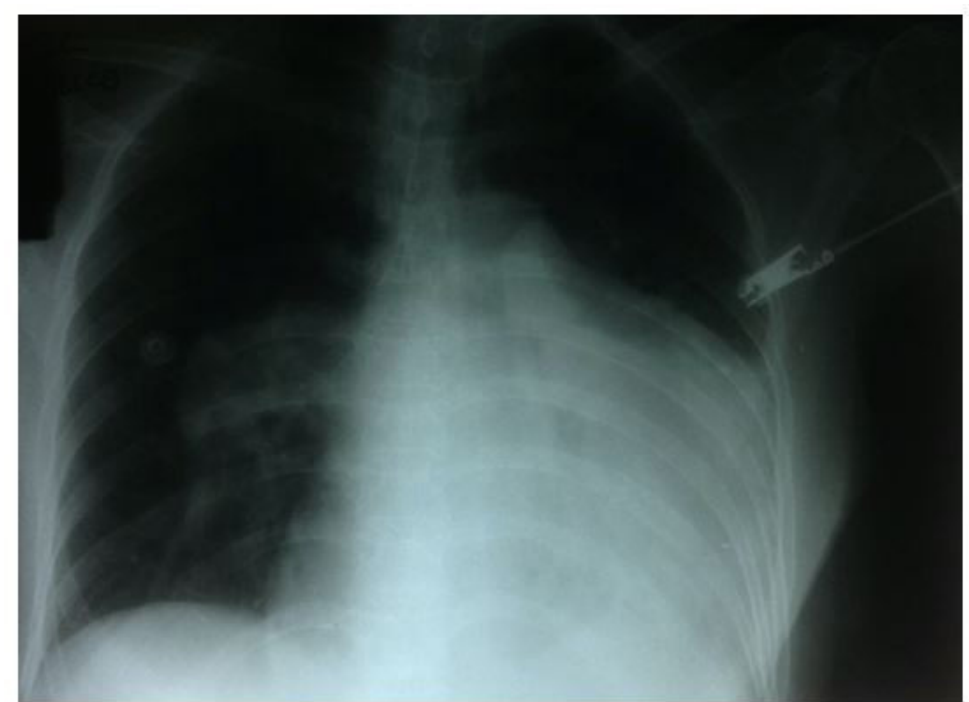

Figura. 1. Radiografía AP de tórax tomada al ingreso. Hay evidencia de cardiomegalia global y además se aprecia una radio-opacidad parahiliar derecha.

Los aneurismas de la arteria pulmonar son raros e infrecuentemente diagnosticados. Generalmente ocurren en un grupo de edad más joven, comparado con los aneurismas aórticos y tienen un incidencia igual por sexos.

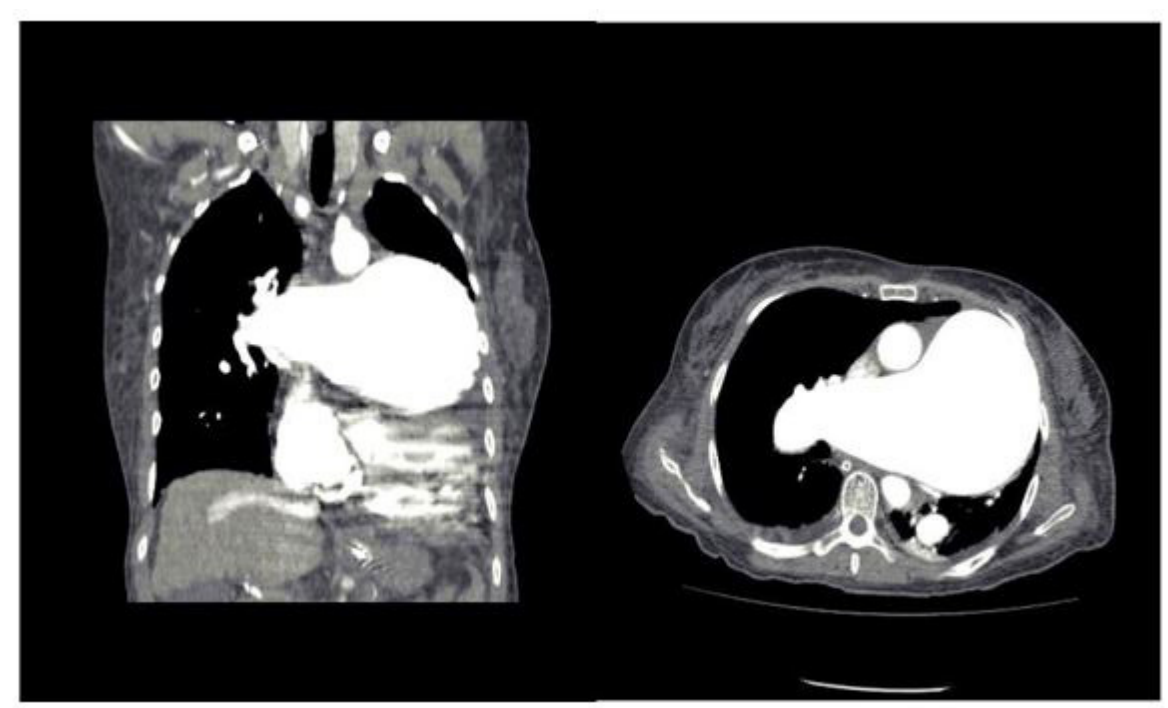

Figura 2. Angiotomografía de tórax. Cortes coronal (derecha) y axial (izquierda) en ventana mediastinal en fase de contraste. Hay dilatación severa del tronco de la arteria pulmonar con $140 \mathrm{~mm}$ en diámetro mayor en corte axial y $121 \mathrm{~mm}$ en diámetro máximo en corte coronal. Además, hay una dilatación de la rama derecha con diámetro máximo con corte axial de $44 \mathrm{~mm}$

La paciente permaneció ocho días en la unidad de cuidado intensivo, donde recibió manejo con antimicrobiano ante la sospecha de enfermedad infecciosa como causante del cuadro de deterioro. No requirió soporte ventilatorio mecánico durante su estancia ni presentó compromiso renal. La analítica de seguimiento se normalizó durante su estancia y los estudios microbiológicos fueron negativos (cuadro1). La 
evolución fue favorable en los días siguientes lográndose el retiro de los medicamentos vasoactivos al quinto día de ingreso y fue trasladada luego de ocho días a sala general de hospitalización.

Finalmente, luego de la evaluación diagnóstica y teniendo en cuenta la respuesta a la fluidoterapia y a los antimicrobianos instaurados se llegó a la conclusión que la causa del cuadro clínico fue infección de posible foco bronquial sin lograr obtener aislamiento microbiológico. El aneurisma de la arteria pulmonar se consideró incidental y además que la sintomatología no era explicada por el mismo, ni que tampoco limitó el manejo terapéutico.

Se planteó entonces realizar estudios de extensión para los hallazgos de la anormalidad del tronco de la arteria pulmonar y de clasificación de la hipertensión pulmonar. Por el tamaño del aneurisma se consideró, y según los estudios complementarios, realizar manejo quirúrgico, sin embargo, la paciente no aceptó y se dio egreso luego de completar el manejo de enfermedades que llevaron a la hospitalización. Ante el alto riesgo de disección de la anormalidad vascular se indicaron controles periódicos con seguimiento ecocardiográfico cada seis meses, los cuales han sido irregulares por la poca adherencia de la paciente.

Cuadro 1. Exámenes analíticos realizados

La definición de los aneurismas de la arteria pulmonar se realiza de acuerdo con sus dimensiones. También pueden clasificarse de acuerdo con la presión intravascular del aneurisma, como de alta y baja presión.

\begin{tabular}{|c|c|c|c|}
\hline Examen & Ingreso & Día 1 & Día 8 \\
\hline $\begin{array}{l}\text { Hemograma } \\
\text {-Leucocitos } \\
\text {-Neutrófilos } \\
\text {-Linfocitos } \\
\text {-Plaquetas } \\
\text {-Hemoglobina } \\
\text {-Hematocrito } \\
\text {-RDW } \\
\text {-VCM } \\
\text {-HCM }\end{array}$ & $\begin{array}{l}15300 \\
53 \% \\
35 \% \\
107000 \\
16,3 \mathrm{gr} / \mathrm{dl} \\
47 \% \\
15,5 \% \\
107 \mathrm{fL} \\
36,3 \mathrm{pg}\end{array}$ & & $\begin{array}{l}8500 \\
80 \% \\
12 \% \\
106000 \\
15,1 \mathrm{gr} / \mathrm{dl} \\
43,6 \% \\
16 \% \\
106 \mathrm{fL} \\
36,6 \mathrm{pg}\end{array}$ \\
\hline Glicemia & 93 mg/dL & & \\
\hline BUN & $11,2 \mathrm{mg} / \mathrm{dl}$ & & $33,5 \mathrm{mg} / \mathrm{dl}$ \\
\hline Creatinina & $0,78 \mathrm{mg} / \mathrm{dl}$ & & $0,98 \mathrm{mg} / \mathrm{dl}$ \\
\hline Baciloscopias & Negativas & & \\
\hline Proteína $\mathrm{C}$ reactiva & $20 \mathrm{mg} / \mathrm{dl}$ & & Menor de $0,5 \mathrm{mg} / \mathrm{dl}$ \\
\hline TP & 18,1 control 13,3 & & 19,6 control 13,3 \\
\hline TPT & 24,5 control 28,1 & & 29 control 28,1 \\
\hline INR & 1,28 & & 1,4 \\
\hline ELISA HIV & Negativo & & \\
\hline VDRL & No reactiva & & \\
\hline $\begin{array}{l}\text { Gases arteriales } \\
-\mathrm{pH} \\
-\mathrm{PCO}_{2} \\
-\mathrm{PO}_{2} \\
-\mathrm{HCO}_{3} \\
\text { - Lactato } \\
\mathrm{BE} \\
\mathrm{Pa} / \mathrm{Fi}\end{array}$ & $\begin{array}{l}7,39 \\
29 \mathrm{~mm} \mathrm{Hg} \\
67 \mathrm{mmHg} \\
17,6 \mathrm{mmol} / \mathrm{l} \\
3,0 \mathrm{mmol} / \mathrm{l} \\
-6,0 \mathrm{mmol} / \mathrm{l} \\
209\end{array}$ & $\begin{array}{l}7,36 \\
30 \mathrm{mmHg} \\
85 \mathrm{mmHg} \\
16,9 \mathrm{mmol} / \mathrm{l} \\
1,8 \mathrm{mmol} / \mathrm{l} \\
-7,4 \mathrm{mmol} / \mathrm{l} \\
265\end{array}$ & $\begin{array}{l}7,40 \\
38 \mathrm{~mm} \mathrm{Hg} \\
88 \mathrm{~mm} \mathrm{Hg} \\
20 \mathrm{mmol} / \mathrm{l} \\
1,03 \mathrm{mmol} / \mathrm{l} \\
2,0 \mathrm{mmol} / \mathrm{l}\end{array}$ \\
\hline TSH & $4,09 \mathrm{uUI} / \mathrm{ml}$ & & \\
\hline $\mathrm{Na}$ & $146 \mathrm{mEq} / \mathrm{l}$ & & \\
\hline K & $4,05 \mathrm{mEq} / \mathrm{l}$ & & \\
\hline
\end{tabular}




\section{Discusión}

Presentamos el caso de una mujer en quien, de manera incidental y sin explicar la sintomatología o curso clínico de la hospitalización, se encontró un aneurisma gigante del tronco de la arteria pulmonar, con datos asociados de hipertensión pulmonar y sin otra causa clara de la anormalidad vascular.

De manera aislada, los aneurismas de la arteria pulmonar tienen una prevalencia estimada de uno por cada 14000 individuos (4). Respecto a los aneurismas aórticos, ocurren en un grupo de edad menor y tienen incidencia similar en ambos sexos (2) (5).

La definición de los aneurismas de la arteria pulmonar se realiza de acuerdo con sus dimensiones: en mujeres un diámetro mayor a 43,4 mm y en hombres un diámetro mayor a 40,4 mm (6). Otros autores sugieren omitir la diferencia por sexo y considerar como límite para definir una aneurisma de la arteria pulmonar principal un diámetro de $29 \mathrm{~mm}$, mientras que para las arterias pulmonares interlobares se considera un límite de $17 \mathrm{~mm}$ (5).

En base a una revisión sistemática reciente también pueden clasificarse de acuerdo con la presión intravascular del aneurisma, como de alta y baja presión (4). En esta

Dentro del mecanismo de formación de los aneurismas de la arteria pulmonar se han considerado múltiples causas. Una de las principales es la hipertensión pulmonar; procesos infecciosos, alteraciones del colágeno, malignidad, entre otras. Más del $50 \%$ de los aneurismas de la arteria pulmonar están asociados a enfermedad cardiaca congénita. revisión se agrupan las de alta presión intravascular dependiendo de su etiología en: asociadas a malformaciones congénitas, idiopáticas y misceláneas. Las de baja presión se catalogan también en tres grupos: asociadas con anormalidades conectivas (infecciosas, genéticas, inflamatorias), idiopáticas y relacionadas a dilatación post estenosis y los aneurismas con dilatación causada por alto flujo a través de la arteria pulmonar como en la regurgitación severa.

Dentro del mecanismo de formación de los aneurismas de la arteria pulmonar se han considerado múltiples causas. Una de las principales es la hipertensión pulmonar (7); no obstante, también se identifican procesos infecciosos, alteraciones del colágeno, malignidad, entre otras (5).

Más del $50 \%$ de los aneurismas de la arteria pulmonar están asociados a enfermedad cardíaca congénita. Los defectos cardíacos asociados con mayor frecuencia corresponden al ductus arterioso persistente, defectos septales ventriculares y defectos septales auriculares $(1,8)$. La valvulopatía aórtica tipo hipoplasia de la válvula aórtica y la válvula aórtica bicúspide también se han identificado dentro de las etiologías (8). La estenosis de la válvula pulmonar se ha descrito como una causa aislada de aneurismas en la circulación pulmonar de manera frecuente (5).

Anteriormente, de los casos relacionados con agentes infecciosos, tuberculosis y sífilis con frecuencia se asociaban a la formación de aneurismas de la arteria pulmonar. Los primeros, por los general, son de tipo intraparenquimatoso (9) y los últimos, en las arterias de gran calibre (10). Hoy en día, las bacterias piógenas son una causa en aumento de formación de pseudoaneurismas (11).

El síndrome de Behcet se asocia a la formación de aneurismas en las arterias del lóbulo inferior derecho, con trombosis recurrentes e inflamación (12). El síndrome de Hughes-Stovin (considerado por algunos como la manifestación cardiovascular del síndrome de Behcet) es un trastorno infrecuente autoinmune que se caracteriza por la formación de aneurisma de la arteria pulmonar, con tromboflebitis recurrente y alto riesgo de ruptura de los aneurismas pulmonares (13). 
Mayo - agosto de 2020 - Pág 149

Las manifestaciones clínicas son inespecíficas e incluso muchos pacientes con aneurismas de diámetros hasta 70 mm permanecen asintomáticos. Los síntomas incluyen disnea, dolor torácico, palpitaciones, sincope y los relacionados a compresión bronquial como tos, aumento de la disnea. La hemoptisis, descrita como un posible síntoma, debe considerarse un signo de alarma de ruptura inminente.
Otras causas menos frecuentes incluyen el trauma penetrante o cerrado, siendo las heridas penetrantes las de mayor frecuencia (5), trauma endovascular asociado a catéteres (14) y los tumores primarios o las metástasis (15) (16).

En muchas ocasiones no se logra establecer una causa para la presencia de dichos aneurismas, estableciéndose así las de tipo idiopático, que de acuerdo a los criterios de Greene y Baldwin pueden definirse así: dilatación de la arteria pulmonar con o sin compromiso del resto del árbol arterial, ausencia de shunts intra o extracardíacos, ausencia de enfermedad pulmonar obstructiva crónica y, ausencia de enfermedad arterial como sífilis o ateromatosis de la vasculatura del árbol pulmonar (17).

En general, las manifestaciones clínicas son inespecíficas e incluso muchos pacientes con aneurismas de diámetros hasta $70 \mathrm{~mm}$ permanecen asintomáticos (5). Los síntomas incluyen disnea, dolor torácico, palpitaciones, sincope y los relacionados a compresión bronquial como tos, aumento de la disnea (5). La hemoptisis, descrita como un posible síntoma, debe considerarse un signo de alarma de ruptura inminente.

El electrocardiograma puede evidenciar signos de hipertrofia auricular o ventricular derecha (18), el ecocardiograma que permite evaluar la función valvular y demostrar la presencia del aneurisma (19) y la angiografía permite evaluar el compromiso de la pared vascular, así como la presión en su interior. La desventaja de este método es su invasividad (20). La tomografía computarizada contrastada confirma el diagnóstico y provee información útil en cuanto al tamaño, localización y extensión, ventajas que también aporta la resonancia magnética, pero sin la exposición a radiación (21).

El manejo puede ser médico o quirúrgico. Se recomienda este último cuando el aneurisma tiene un tamaño mayor a $6 \mathrm{~cm}$ o si es sintomático sin importar su tamaño, ya que es mayor el riesgo de ruptura en estos casos. La American Heart Association considera un diámetro $>5.5 \mathrm{~cm}$ de acuerdo con la experiencia obtenida con enfermedad aórtica (5).

El tratamiento conservador se indica en pacientes asintomáticos y en quienes el aneurisma no supera el tamaño de $6 \mathrm{~cm}$ y se contraindica en casos con hipertensión pulmonar asociada o enfermedades valvulares, pues se asocian a estrés hemodinámico persistente que puede conllevar a mayor dilatación (22). Estos pacientes requieren ser re-evaluados constantemente y considerar manejo quirúrgico en caso de compresión de estructuras adyacentes, formación de trombos en el saco aneurismático, incremento $>5 \mathrm{~mm}$ de diámetro aneurismático en seis meses, la aparición de síntomas o la evidencia de patología valvular (23).

La disección de un aneurisma de la arteria pulmonar es una complicación que ocurre en aproximadamente en $19 \%$ de los pacientes con aneurisma y sin hipertensión pulmonar (24). La localización más frecuente de disección corresponde al tronco principal de la arteria pulmonar ( $80 \%$ ) y únicamente un $15 \%$ de todas las disecciones son diagnosticadas en pacientes vivos. Los síntomas de presentación incluyen disnea, dolor retroesternal, cianosis, choque cardiogénico y muerte súbita. El taponamiento cardíaco resultante de la disección del aneurisma se ha identificado como la principal causa de muerte (5).

Recientemente Gupta et al. (25) sugieren un algoritmo de manejo de este grupo de pacientes y dada su practicidad nos parece importante tenerlo presente en la práctica clínica rutinaria (figura 3). 
El manejo puede ser médico o quirúrgico. Se recomienda este último cuando el aneurisma tiene un tamaño mayor a $6 \mathrm{~cm}$ o si es sintomático sin importar su tamaño, ya que es mayor el riesgo de ruptura en estos casos.

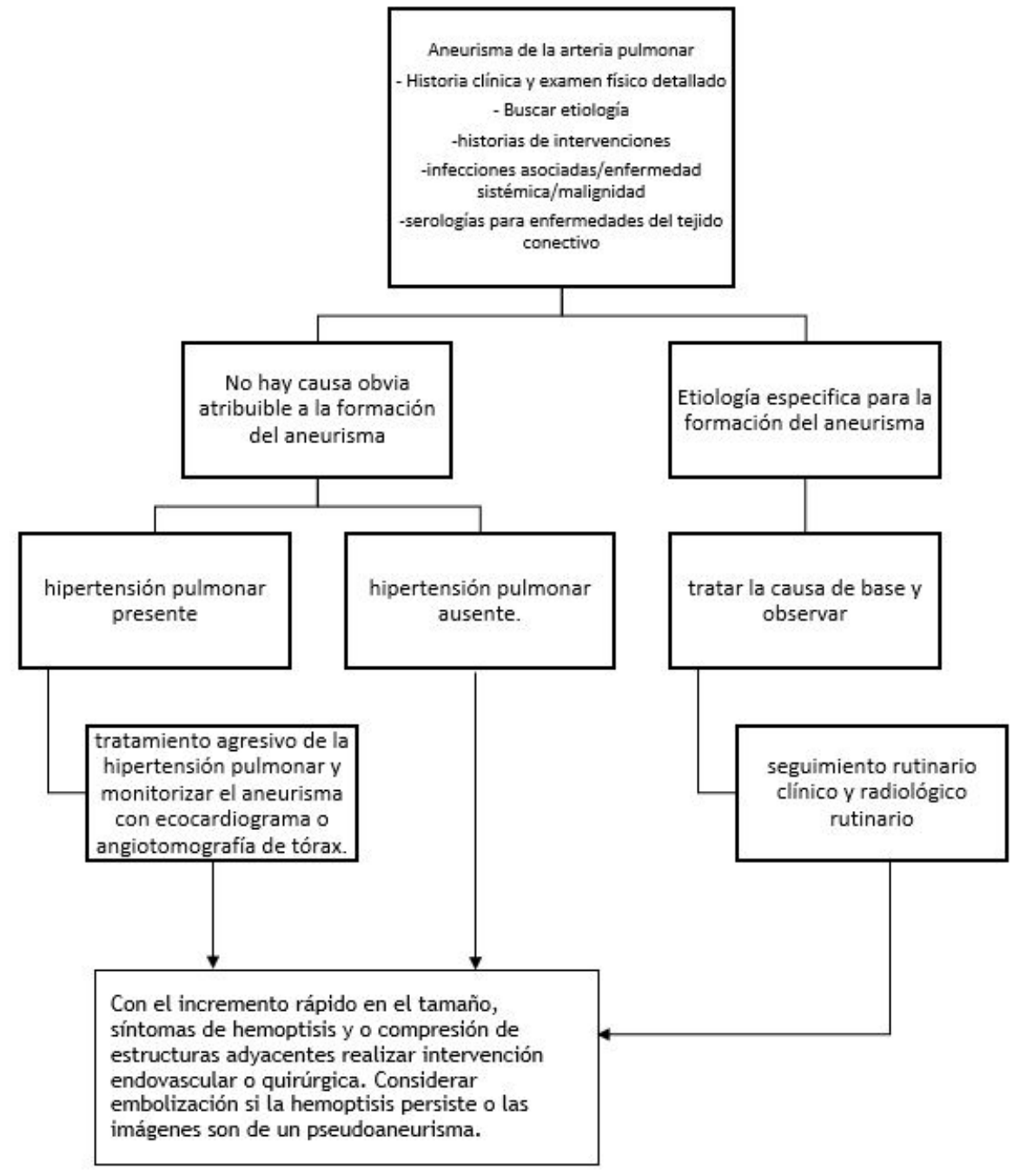

\section{Conclusión}

Los aneurismas de la arteria pulmonar no se presentan con síntomas característicos o distintivos que permitan su diagnóstico claro, por lo que rara vez se diagnostican basados en sus manifestaciones. El incremento en el uso de la tomografía computarizada ha aumentado la detección de esta anormalidad; sin embargo, a la fecha no se cuenta con una guía de diagnóstico, manejo o seguimiento de estos pacientes. Se debe buscar un consenso que pueda estandarizar criterios diagnósticos, el uso de imágenes de seguimiento y las estrategias de manejo.

\section{Conflictos de intereses}

Los autores manifiestan no tener conflictos de intereses. 


\section{Bibliografía}

1. Deterling RA, Clagett OT. Aneurysm of the pulmonary artery; review of the literature and report of a case. Am Heart J. 1947 Oct;34 (4):471-99.

2. Blades B, Ford W, Clark P. Pulmonary artery aneurysms; report of a case treated by surgical intervention. Circulation. 1950 0ct;2 (4):565-71.

3. Metras D, Ouattara K, Quezzin-Coulibaly A. Aneurysm of the pulmonary artery with cystic medial necrosis and massive pulmonary valvular insufficiency. Report of two successful surgical cases. Eur J Cardio-Thorac Surg Off J Eur Assoc Cardio-Thorac Surg. 1987;1 (2):119-24.

4. Duijnhouwer AL, Navarese EP, Van Dijk APJ, Loeys B, Roos-Hesselink JW, De Boer MJ. Aneurysm of the Pulmonary Artery, a Systematic Review and Critical Analysis of Current Literature. Congenit Heart Dis. 2016 Apr;11 (2):102-9.

5. Kreibich M, Siepe M, Kroll J, Höhn R, Grohmann J, Beyersdorf F. Aneurysms of the pulmonary artery. Circulation. 2015 Jan 20;131 (3):310-6.

6. Truong QA, Massaro JM, Rogers IS, Mahabadi AA, Kriegel MF, Fox CS, et al. Reference values for normal pulmonary artery dimensions by noncontrast cardiac computed tomography: the Framingham Heart Study. Circ Cardiovasc Imaging. 2012 Jan;5 (1):147-54.

7. Boerrigter B, Mauritz G-J, Marcus JT, Helderman F, Postmus PE, Westerhof N, et al. Progressive dilatation of the main pulmonary artery is a characteristic of pulmonary arterial hypertension and is not related to changes in pressure. Chest. 2010 Dec;138 (6):1395-401.

8. Bartter T, Irwin RS, Nash G. Aneurysms of the pulmonary arteries. Chest. 1988 Nov;94 (5):1065-75.

9. Plessinger VA, Jolly PN. Rasmussen's aneurysms and fatal hemorrhage in pulmonary tuberculosis. Am Rev Tuberc. 1949 Nov;60 (5):589-603, illust.

10. Paulo N, Cascarejo J, Vouga L. Syphilitic aneurysm of the ascending aorta. Interact Cardiovasc Thorac Surg. 2012 Feb;14 (2):223-5.

11. Kim HS, Oh Y-W, Noh HJ, Lee KY, Kang E-Y, Lee SY. Mycotic pulmonary artery aneurysm as an unusual complication of thoracic actinomycosis. Korean J Radiol. 2004 Mar;5 (1):68-71.

12. Kohno S, Fujikawa M, Kanda T, Asai S, Hirota M, Sameshima Y. A case of Behçet's syndrome with rupture of a pulmonary aneurysm: autopsy findings and a literature review. Jpn J Med. 1986 Aug;25 (3):293-300.

13. Chalazonitis AN, Lachanis SB, Mitseas P, Argyriou P, Tzovara J, Porfyrides $P$, et al. Hughes-Stovin syndrome: a case report and review of the literature. Cases J. 2009 Jan 29;2:98. 
14. Boyd KD, Thomas SJ, Gold J, Boyd AD. A prospective study of complications of pulmonary artery catheterizations in 500 consecutive patients. Chest. 1983 Sep;84 (3):245-9.

15. Shum PL, Ngo B, Chen X, Jarvis R. Pulmonary artery pseudoaneurysm secondary to metastatic breast cancer. South Asian J Cancer. 2018;7 (1):20.

16. Agarwal PP, Dennie CJ, Matzinger FR, Peterson RA, Seely JM. Pulmonary artery pseudoaneurysm secondary to metastatic angiosarcoma. Thorax. 2006 Apr;61 (4):366.

17. Greene DG, Baldwin ED. Pure congenital pulmonary stenosis and idiopathic congenital dilatation of the pulmonary artery. Am J Med. 1949 Jan;6 (1):24-40.

18. Shankarappa RK, Moorthy N, Chandrasekaran D, Nanjappa MC. Giant pulmonary artery aneurysm secondary to primary pulmonary hypertension. Tex Heart Inst J. 2010;37 (2):244-5.

19. Seguchi M, Wada H, Sakakura K, Kubo N, Ikeda N, Sugawara Y, et al. Idiopathic pulmonary artery aneurysm. Circulation. 2011 Oct 4;124 (14):e369-370.

20. Tsui EY, Cheung YK, Chow L, Chau LF, Yu SK, Chan JH. Idiopathic pulmonary artery aneurysm: digital subtraction pulmonary angiography grossly underestimates the size of the aneurysm. Clin Imaging. 2001 Jun;25 (3):178-80.

21. Geiger J, Hirtler D, Bürk J, Stiller B, Arnold R, Jung B, et al. Postoperative pulmonary and aortic 3D haemodynamics in patients after repair of transposition of the great arteries. Eur Radiol. 2014 Jan;24 (1):200-8.

22. Veldtman GR, Dearani JA, Warnes CA. Low pressure giant pulmonary artery aneurysms in the adult: natural history and management strategies. Heart $\mathrm{Br}$ Card Soc. 2003 Sep;89 (9):1067-70.

23. Vural AH, Türk T, Ata Y, Göncü T, Ozyazicioglu A. Idiopathic asymptomatic main pulmonary artery aneurysm: surgery or conservative management? A case report. Heart Surg Forum. 2007;10 (4):E273-275.

24. Inayama Y, Nakatani Y, Kitamura H. Pulmonary artery dissection in patients without underlying pulmonary hypertension. Histopathology. 2001 May;38 (5):435-42.

25. Gupta M, Agrawal A, lakovou A, Cohen S, Shah R, Talwar A. Pulmonary artery aneurysm: a review. Pulm Circ. 2020 Mar;10 (1):2045894020908780. 